Communications in Physics, Vol. 23, No. 2 (2013), pp. 139-146

\title{
MAGNETIC PROPERTIES AND GIANT MAGNETOCALORIC EFFECT IN Mn-BASED HEUSLER COMPOUNDS
}

\author{
NGUYEN HUY DAN \\ Institute of Materials Science, \\ Vietnam Academy of Science and Technology, \\ 18 Hoang Quoc Viet, Cau Giay, Hanoi, Vietnam \\ NGUYEN MANH AN \\ Hong Duc University, 565 Quang Trung, Dong Ve, Thanh Hoa \\ Received 02 April 2013; revised manuscript received 31 May 2013 \\ Accepted for publication 10 June 2013
}

\begin{abstract}
Magnetic properties and giant magnetocaloric effect (GMCE) of Mn-based Heusler compounds such as Co-Mn-Si, Ni-Mn-Sn, Ni-Mn-Sb have been investigated. The results show that the structure strongly influences on magnetic properties and GMCE of these alloys. The coexistence of ferromagnetic (FM) and antiferromagnetic (AFM) orders is observed. The magnetic phase transitions can be controlled by changing composition and annealing condition of the alloys. GMCEs with large magnitude and wide working temperature range have been obtained on these alloys showing their application potential for magnetic refrigeration technology.
\end{abstract}

\section{INTRODUCTION}

Heusler alloys have been attracting a lot of scientists by virtue of their giant magnetocaloric effect (GMCE) and application potential for magnetic refrigeration [1-8]. The magnetic refrigeration bases on the principle of magnetic entropy change of the materials under variation of magnetic field. The application of magnetocaloric materials in the refrigerators has the advantages of avoiding the environmental pollution (unlike the refrigerators using compression gases), improving the cooling efficiency (saving energy), reducing noise and fitting to some special cases. The main problems to be addressed to improve the practical applications of the magnetocaloric materials are: i) creating GMCE in low field, because it is very difficult to create a large magnetic field in the home appliances; ii) taking the magnetic phase transition temperature (working temperature) of materials with GMCE to the room temperature; iii) extending the working temperature range (range with GMCE) for materials to be cooled in a large temperature range. In addition, some other properties of materials such as heat capacity, electrical conductivity, thermal conductivity, durability, price... are also concentrated for the application of the GMCE materials. The reported results showed that both the positive (inverse) and negative (normal) GMCEs could be observed in Heusler alloys by changing composition and fabrication conditions. The coexistence of ferromagnetic (FM) and antiferromagnetic (AFM) orders was also observed in these alloys. Changing composition and fabrication 
conditions is a common way to understand the magnetic mechanism and achieve the desired GMCEs for the alloys [8-11]. The magnetic orders and the magnetocaloric effects in Heusler alloys were found to be very sensitive to their composition and fabrication conditions. Therefore, detailed and systematic studies on these alloys are still needed.

In this paper, we summarize our results of investigation of magnetic and magnetocaloric properties of Mn-based Heusler alloys including Co-Mn-Si, Ni-Mn-Sn and Ni$\mathrm{Mn}-\mathrm{Sb}$ prepared by means of arc-melting and subsequently annealing.

\section{EXPERIMENTAL}

The samples were prepared by arc-melting method from pure elements $(>3 N)$ and some of them were then annealed at various annealing temperatures and time in vacuum. Because $\mathrm{Mn}$ is evaporated during arc-melting, an excess of $15 w t \%$ of $\mathrm{Mn}$ was added to the nominal compositions. This compensation amount of Mn was determined basing on thoroughly investigations of the loss of $3 w t \%$ of Mn after each time of arc-melting in the same preparation conditions and each sample was arc-melted five times. The structure of the samples were analyzed by X-ray diffraction (XRD) using a Siemens D5000 X-Ray diffractometer with $\mathrm{CuK}_{\alpha}$ radiation $(\lambda=1.54056 \AA)$. Isothermal magnetization curves and thermomagnetization curves were measured by Vibrating Sample Magnetometer (VSM). The experimental data of magnetization are used to calculate magnetic entropy change $\Delta S_{m}$ using Maxwell's relations:

$$
\Delta S_{m}=\int_{H_{1}}^{H_{2}}\left(\frac{\partial M}{\partial T}\right)_{H} d H
$$

\section{RESULTS AND DISCUSSION}

Some recent studies showed that Co-Mn-Si compounds could exhibit a positive GMCE between the low-temperature AFM and high-temperature FM phase transitions. The substitution of Si by Ge in the CoMnSi alloy might cause a change of unit cell volume and a change in the relative stability of AFM and FM ordering of $\mathrm{CoMnSi}_{1-x} \mathrm{Ge}_{x}$ alloys [12]. The magnetic phase transitions in the CoMnSi system is highly sensitive to the separation of Mn atoms in the lattice [13]. Study on the substitution of Mn by Fe in the $\mathrm{CoMn}_{0.95} \mathrm{Fe}_{0.05} \mathrm{Si}$ alloy indicated that MCE is greatly enhanced at low magnetic field below $20 \mathrm{kOe}[14]$. In this study we investigate structure, magnetic properties and magnetocaloric effect of $\mathrm{CoMn}_{1-x} \mathrm{Fe}_{x} \mathrm{Si}(\mathrm{x}=0,0.05,0.1,0.15$ and 0.2) compounds. XRD patterns of the $\mathrm{CoMn}_{1-x} \mathrm{Fe}_{x} \mathrm{Si}$ samples are shown in Fig. 1. We can see that all of diffraction peaks in XRD patterns of the samples with $\mathrm{x}=0$ coincide with ones of standard XRD pattern of the CoMnSi alloy. Thus, we could conclude that these samples are single phase of the half Heusler CoMnSi. The XRD patterns of the as-melted samples with $\mathrm{x}=0.05,0.1$ and 0.15 have some diffraction peaks corresponding to diffraction peaks of other phases of $\mathrm{Co}_{2} \mathrm{MnSi}$, $\mathrm{CoFe}, \mathrm{FeSi}, \mathrm{Mn}$ and Fe besides the diffraction peaks of CoMnSi phase. However, number and intensity of diffraction peaks corresponding to CoMnSi phase are greater than those of other phases. This proves the CoMnSi phase has a high volume fraction in the samples. As for the sample with $x=0.2$, diffraction peaks corresponding to the $\mathrm{Co}_{2} \mathrm{MnSi}$ phase 
are dominated. After annealing at $850^{\circ} \mathrm{C}$ for $60 \mathrm{~h}$, a number of the unexpected phases is decreased in the samples with $x=0.05,0.1,0.15$. This means that annealing process has affected the crystallization of the samples and makes the structure of them are more stable. This result is consistent with K. Morrison's result et al [13]. Note that intensity of diffraction peaks is only comparable each other in the same pattern.

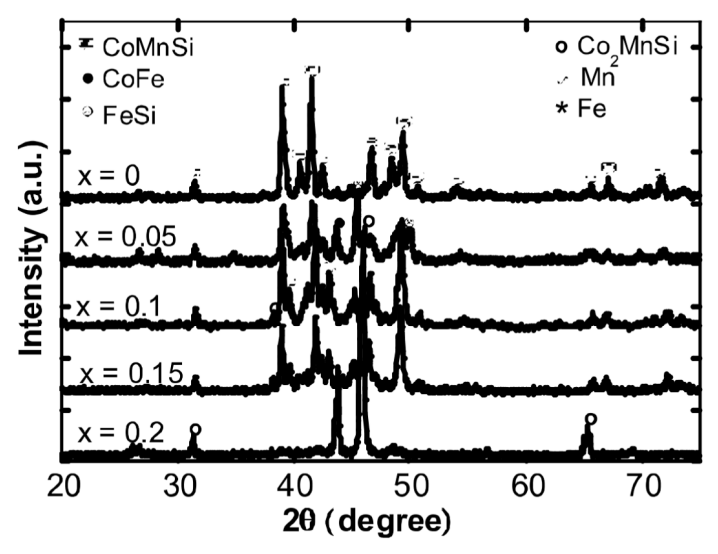

(a)

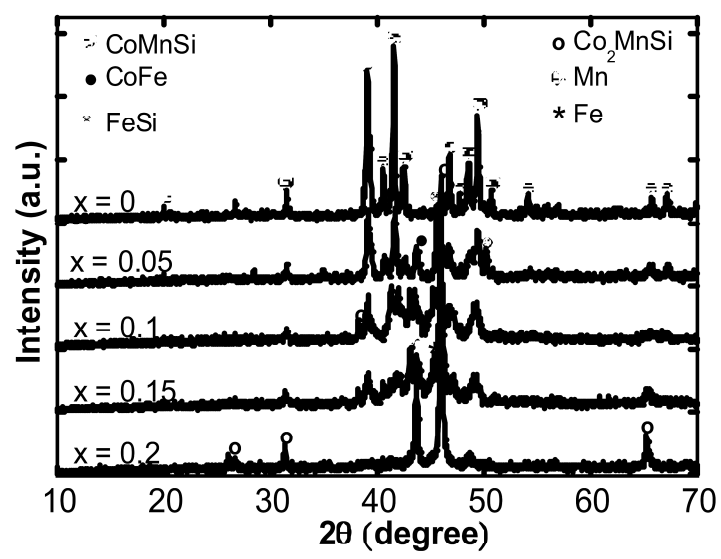

(b)

Fig. 1. XRD patterns of the $\mathrm{CoMn}_{1-x} \mathrm{Fe}_{x} \mathrm{Si}$ samples before (a) and after annealing at $850^{\circ} \mathrm{C}$ for $60 \mathrm{~h} \mathrm{(b)}$.

The magnetic hysteresis measurements (Fig. 2a) show that all of the $\mathrm{CoMn}_{1-x} \mathrm{Fe}_{x} \mathrm{Si}$ samples are soft magnetic with coercivity less than 100 Oe. Their saturation magnetization is raised with increasing concentration of Fe. It should be noted from Fig. $2 \mathrm{~b}$ that the annealed sample $(\mathrm{x}=0)$ reveals AFM behavior We can see the magnetic phase transition from AFM at low magnetic field to FM at higher magnetic field. Under an applied field of 500 Oe, this AFM-FM phase transition happen at a temperature $T_{M}=365 \mathrm{~K}$ (see inset in Fig. 2b). The values of magnetic entropy change $\left(\Delta S_{m}\right)$ of the alloys in as-melted and annealed states can be calculated from the experimental data isothermal magnetization (Fig. 3a) by using the Eq. (1) $\Delta S_{m}$ versus temperature of the alloys in the as-melted and annealed sample with $\mathrm{x}=0$ is plotted in Fig. 3b. The as-melted alloy has only negative $\Delta S_{m}$ while the annealed alloy has both of the negative and positive $\Delta S_{m}$. The peaks of the entropy change appear just around $T_{C}$ and $T_{M}$ corresponding to the FM-PM and AFM-FM phase transition, respectively. Maximum $\Delta S_{m}$ under a magnetic field change of $12 \mathrm{kOe}$ is about $-0.6 \mathrm{~J} / \mathrm{kg} . \mathrm{K}$ at $385 \mathrm{~K}$ for the as-melted alloy, and $-0.6 \mathrm{~J} / \mathrm{kg} . \mathrm{K}$ at $371 \mathrm{~K}$ and $0.3 \mathrm{~J} / \mathrm{kg} . \mathrm{K}$ at $303 \mathrm{~K}$ for the annealed alloy. Our experiments showed that the AFM order was not observed in other annealed alloys but only in the sample with $x=0$. This AFM order was disappeared after replacing a small Fe content $(x=0.05)$ for $\mathrm{Mn}$ in to the CoMnSi-based alloy as shown in the inset of Fig. 2b. We also tried to investigate the $\mathrm{CoMn}_{1-x} \mathrm{Fe}_{x} \mathrm{Si}$ alloys with smaller concentrations of $\mathrm{Fe}(x=0.01-0.04)$ to find a critical of Fe-concentration for the AFMFM transition. However, the AFM order was not 
observed clearly in these samples. This phenomenon has been explained as a result of the stamping out the AFM states in this sample by Fe. This means the replacement of a small Mn content by Fe affects a strongly change in magnetic orders and strengthens the FM order of the alloys. Further, Curie temperature of the $\mathrm{CoMn}_{1-x} \mathrm{Fe}_{x} \mathrm{Si}$ alloys almost shifts to higher temperature when Fe-concentration increases.

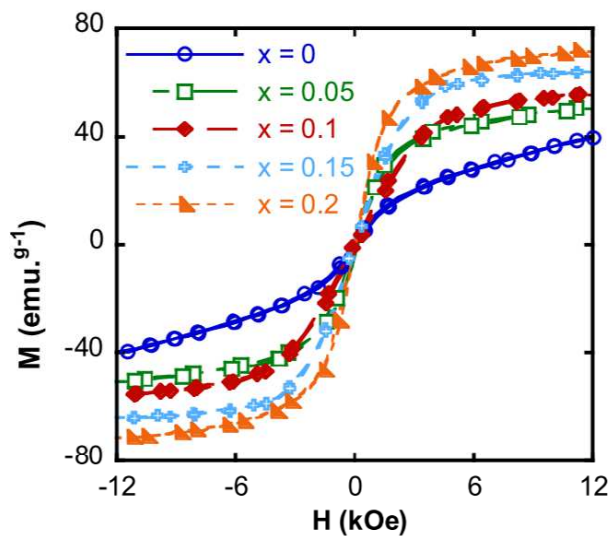

(a)

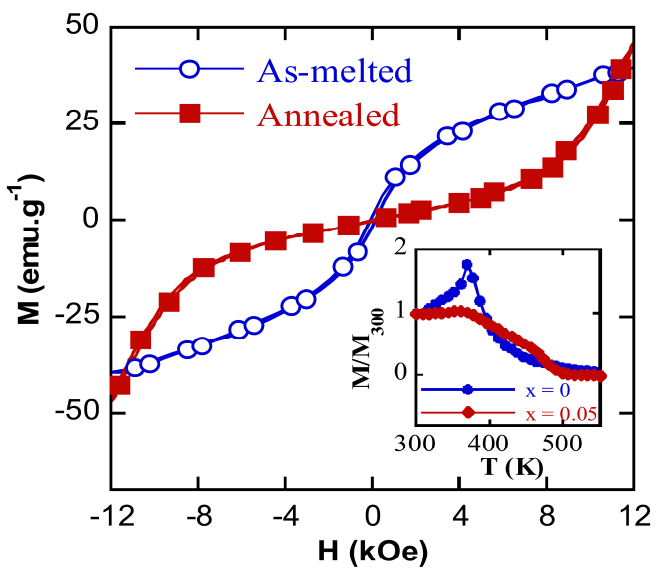

(b)

Fig. 2. Hysteresis loops of the as-melted $\mathrm{CoMn}_{1-x} \mathrm{Fe}_{x} \mathrm{Si}$ samples (a) and the sample with $x=0$ annealed at $850^{\circ} \mathrm{C}$ for $60 \mathrm{~h} \mathrm{(b).} \mathrm{The} \mathrm{inset} \mathrm{shows} \mathrm{thermomag-}$ netization curves in an applied field of 500 Oe of the annealed samples with $x=0$ and $x=0.05$.

The AFM-FM phase transition could be found in other Heusler alloys with various compositions and fabrication conditions [15-18]. Therefore, we have tried to search for this magnetic phase transition, which relates to the positive GMCE, in Ni-Mn-Sn and Ni$\mathrm{Mn}-\mathrm{Sb}$ alloys. Fig. 4 presents thermomagnetization curves under an applied field of 100 Oe of the as-melted and annealed (at $1000^{\circ} \mathrm{C}$ for $2 \mathrm{~h}$ ) $\mathrm{Ni}_{0.5} \mathrm{Mn}_{0.5-x} \mathrm{Sn}_{x}(\mathrm{x}=0-0.4$ ) alloys. We can see the magnetization and magnetic order strongly depend on Sn concentration. The FM order is main phase with an FM-PM phase transition in a range of temperature from $\sim 300$ to $\sim 360 \mathrm{~K}$, depending on Sn-concentration Particularly, with further decrease of temperature, an AFM type transition was observed in $M(T)$ curves of the samples with $x=0.13,0.14$ and 0.15 . That means both of the AFM and FM order can exist in the alloy with appropriate Sn-concentrations. The unclear AFM orders are observed in the as-melted alloys. However, these AFM order become more visible in the annealed alloys. The AFM-FM phase transition temperature shifts toward low temperature when Sn-concentration increases. Thermomagnetization curves in various magnetic fields of the sample with $x=13\left(\mathrm{Ni}_{0.5} \mathrm{Mn}_{0.37} \mathrm{Sn}_{0.13}\right)$ were measured systematically and are plotted in Fig. 5a. This result indicates that a quite strong AFM coupling exists in the alloy, which is maintained until an external magnetic field up to $12 \mathrm{kOe}$. 


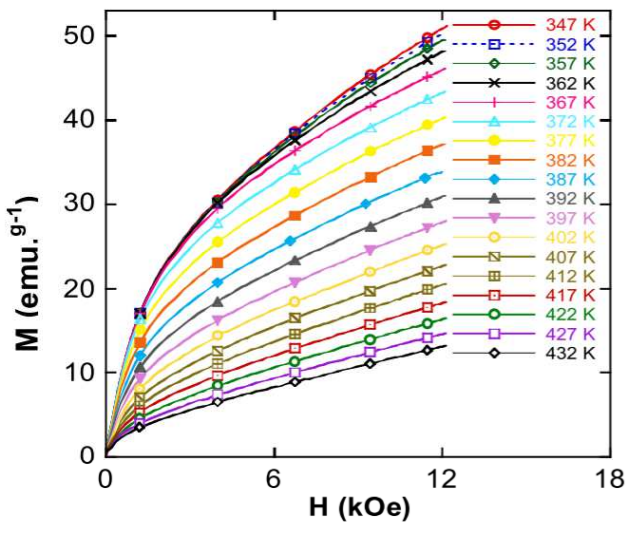

(a)

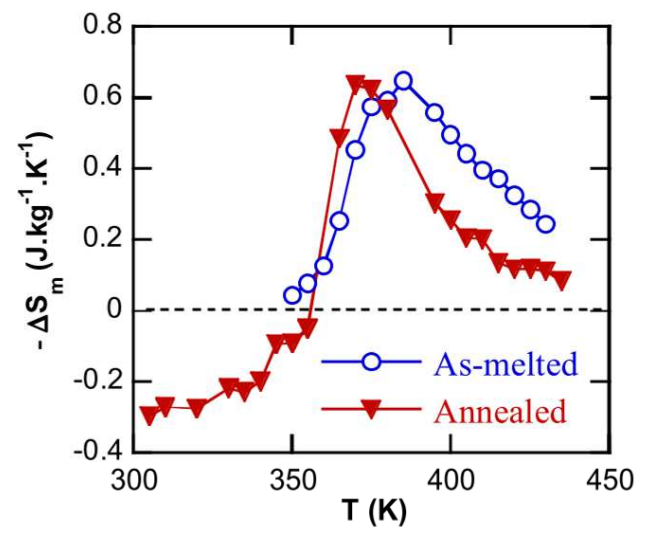

(b)

Fig. 3. Isothermal magnetization curves of CoMnSi $(x=0)$ sample in as-melted state (a) and its magnetic entropy change vs temperature in as-melted and annealed states (b).

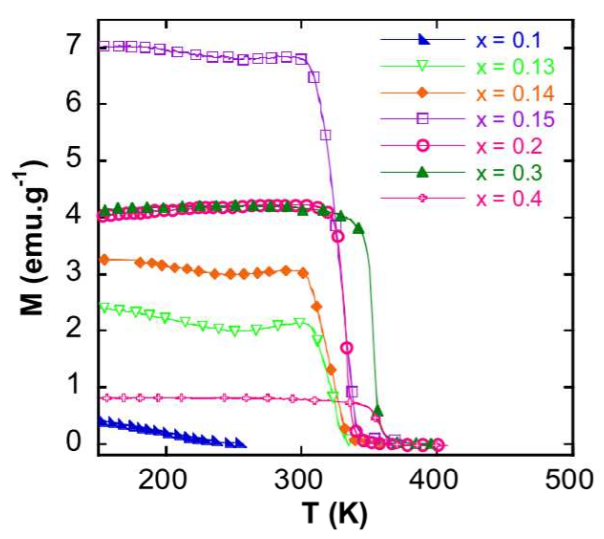

(a)

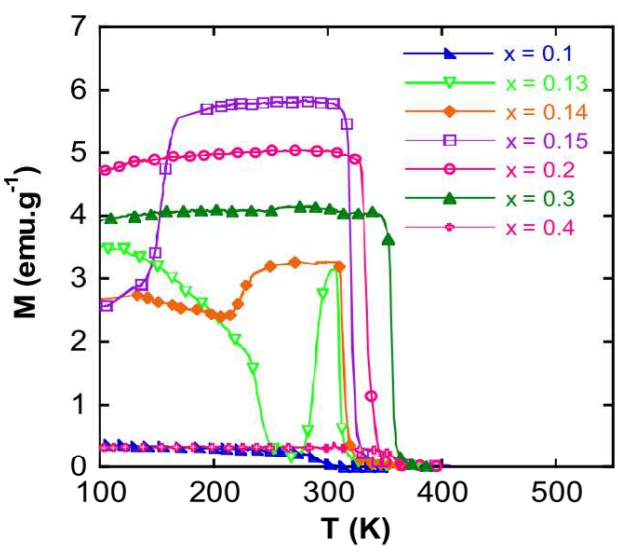

(b)

Fig. 4. Themomagnetization curves in an applied field of 100 Oe of $\mathrm{Ni}_{0.5} \mathrm{Mn}_{0.5-x} \mathrm{Sn}_{x}(\mathrm{x}=0-0.4)$ alloys before and after annealing at $1000^{\circ} \mathrm{C}$ for $2 \mathrm{~h}$.

The magnetic entropy change, $\Delta \mathrm{S}_{m}$ of the annealed $\mathrm{Ni}_{0.5} \mathrm{Mn}_{0.5-x} \mathrm{Sn}_{x}$ were canculated and plotted in Fig. 5b. Here, the magnetic field was changed from 0 to $12 \mathrm{kOe}$. The inset of Fig. 5b shows $\Delta \mathrm{S}_{m}(\mathrm{~T})$ for the sample with $\mathrm{x}=0.13$. We can see that a peak of the entropy change appears around $310 \mathrm{~K}$ corresponding to the FM-PM transition and the other around $290 \mathrm{~K}$ corresponding to the AFM-FM transition. Maximum $\Delta \mathrm{S}_{m}$ values under a field change of $12 \mathrm{kOe}$ are $1.8 \mathrm{~J} / \mathrm{kg} . \mathrm{K}$ at $290 \mathrm{~K}$ and $-1.5 \mathrm{~J} / \mathrm{kg} . \mathrm{K}$ at $31 \mathrm{~K}$, which are quite large in comparison with those of other MCE materials. 


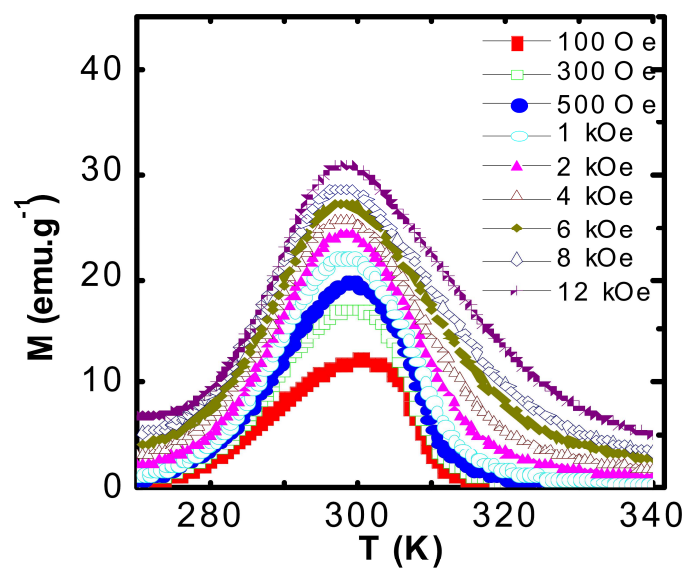

(a)

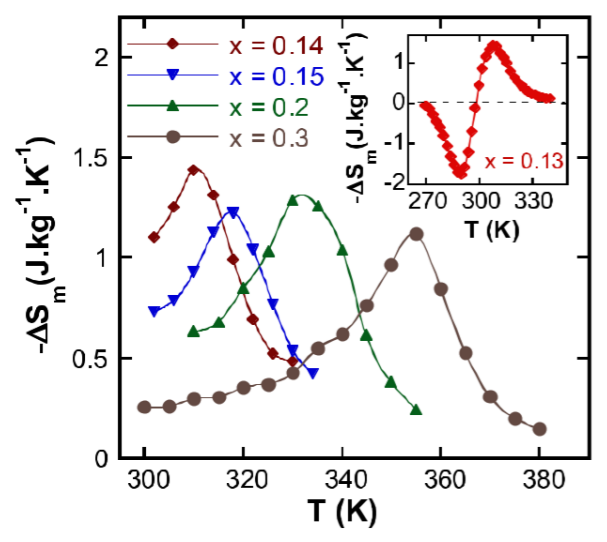

(b)

Fig. 5. $\mathrm{M}(\mathrm{T})$ curves in various applied field of $\mathrm{Ni}_{0.5} \mathrm{Mn}_{0.5-x} \mathrm{Sn}_{x}$ alloy with $\mathrm{x}=$ 0.13 (a) and $\Delta \mathrm{S}_{m}(\mathrm{~T})$ for various $\mathrm{x}(\mathrm{b})$. The inset shows $\Delta \mathrm{S}_{m}(\mathrm{~T})$ for $\mathrm{x}=0.13$. All the samples were annealed at $1000^{\circ} \mathrm{C}$ for $2 \mathrm{~h}$.

As for the $\mathrm{Ni}_{0.5} \mathrm{Mn}_{0.5-x} \mathrm{Sb}_{x}$ alloys, no AMF-FM transition was observed in both the as-quenched and annealed samples with $x=0.1,0.2,0.3$ and 0.4. Fig. 6 shows hysteresis loops at room temperature and thermomagnetization curves in an applied field of $12 \mathrm{kOe}$ of the $\mathrm{Ni}_{0.5} \mathrm{Mn}_{0.5-x} \mathrm{Sb}_{x}$ alloys with $x=0.1,0.2,0.3$ and 0.4 after annealing at $1000^{\circ} \mathrm{C}$ for $2 \mathrm{~h}$. We can see that the saturation magnetization and the Curie temperature, $T_{C}$, of the alloy strongly depends on Sb-concentration. The saturation magnetization first increases and then decreases with increasing $\mathrm{x}$ from 0.1 to 0.4 , while the $T_{C}$ monotonically increases. It should be noted that the magnetic phase transition width clearly varies with $x$. This probably is due to the multi-phase structure of the samples. In order to compare with the $\mathrm{Ni}_{0.5} \mathrm{Mn}_{0.5-x} \mathrm{Sn}_{x}$ alloys, the magnetic properties of the $\mathrm{Ni}_{0.5} \mathrm{Mn}_{0.5-x} \mathrm{Sb}_{x}$ samples with $x=0.11,0.12,0.13,0.14$ and 0.15 were also investigated. The magnetic hysteresis measurement indicates that all the as-melted and annealed alloys are soft magnetic. The results of thermomagnetization measurements in an applied magnetic field of $12 \mathrm{kOe}$ of these alloys are presented in Fig. 7a Both the saturation magnetization and the Curie temperature of the alloy gradually increase with increasing $\mathrm{x}$ in the range of $0.11-0.15$. An unusual phenomenon is observed in $\mathrm{Ni}_{0.5} \mathrm{Mn}_{0.38} \mathrm{Sb}_{0.12}$ at temperature around $320 \mathrm{~K}$ There is a small peak in the $M(T)$ curves corresponding to an AFM order. This peak becomes more visible in the inset of Fig. 7a This observation may be a stimulation for further studying the AFM-FM transition in this Heusler alloy. $\Delta \mathrm{S}_{m}(\mathrm{~T})$ of the samples with $\mathrm{x}=0.2$ and 0.3 were calculated and plotted in Fig. $7 \mathrm{~b}$. We can realize that GMCE of these alloys is quite large. 


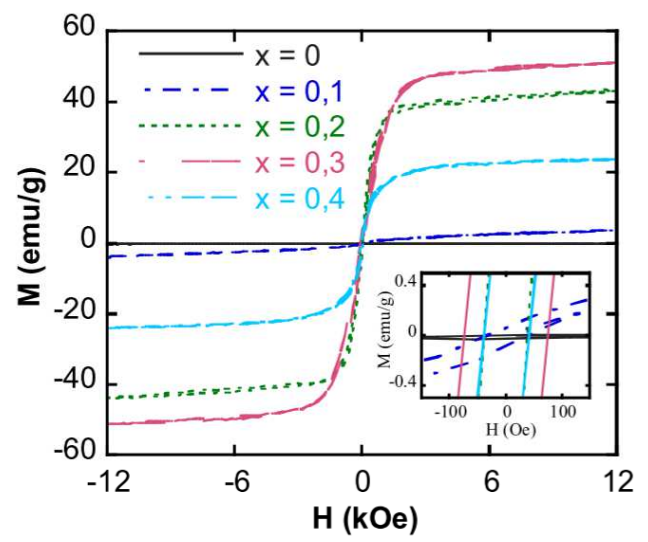

(a)

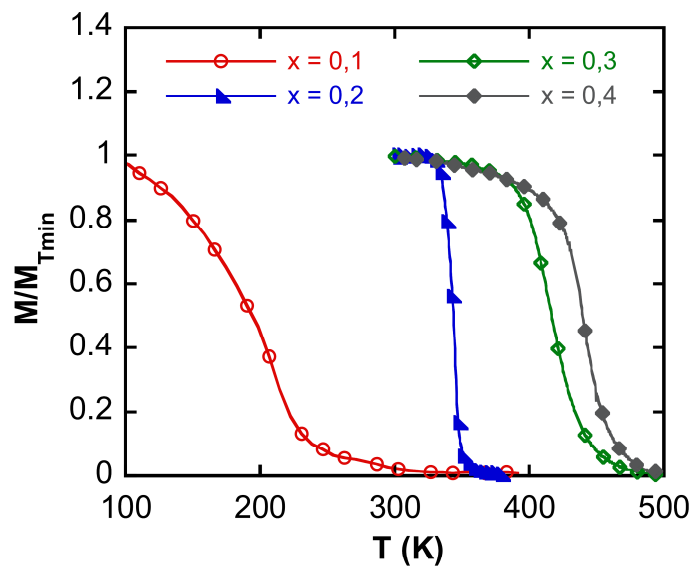

(b)

Fig. 6. Hysteresis loops at room temperature (a) and thermomagnetization curves in an applied field of $500 \mathrm{Oe}(\mathrm{b})$ of the $\mathrm{Ni}_{0.5} \mathrm{Mn}_{0.5-x} \mathrm{Sb}_{x}$ samples annealed at $1000^{\circ} \mathrm{C}$ for $2 \mathrm{~h}$. The inset is an enlargement of the $\mathrm{M}(\mathrm{H})$ curves at low magnetic field.

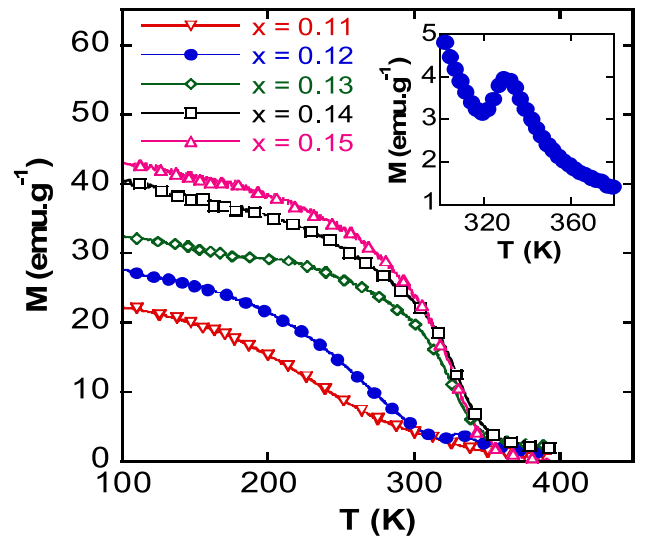

(a)

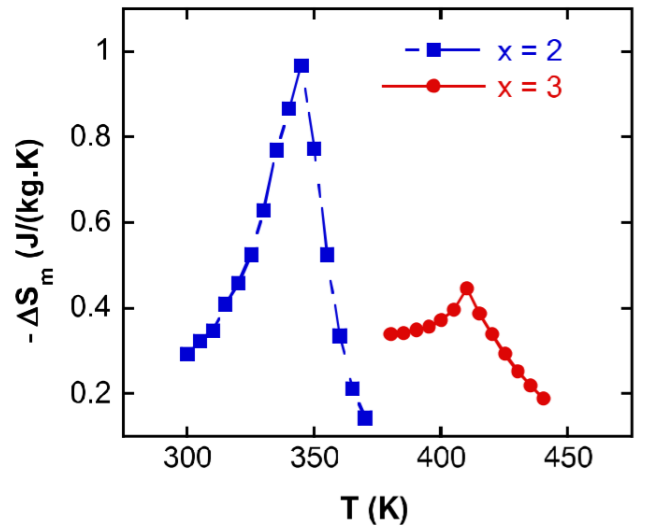

(b)

Fig. 7. Themomagnetization curves in an applied field of $12 \mathrm{kOe}$ of the annealed $\mathrm{Ni}_{0.5} \mathrm{Mn}_{0.5-x} \mathrm{Sb}_{x}$ alloys with $x=0.11-0.15$ (a) and $\Delta S_{m}(\mathrm{~T})$ of the samples with $x=0.2$ and 0.3 . The inset shows AFM-FM transition of the alloy with $x=0.12$.

\section{CONCLUSION}

Magnetic properties and giant magnetocaloric effect (GMCE) of Mn-based Heusler compounds of Co-Mn-Si, Ni-Mn-Sn and Ni-Mn-Sb have been investigated systematically. Antiferromagnetic- ferromagnetic transition is observed in these Heusler alloys by varying composition and annealing condition. Both the positive and negative giant magnetocaloric 
effects can be obtained in these alloys. The magnetic phase transitions, i.e. giant magnetocaloric effect, of the alloys can be controlled in room temperature region to make them possible for application in magnetic refrigeration technology.

\section{ACKNOWLEDGMENTS}

This work was supported by the National Foundation for Science and Technology

Development (NAFOSTED) under grant number of 103.02-2011.23 and the Ministry of Education and Training under project number B2013-42-24 A part of the work was done in the Key Laboratory for Electronic Materials and Devices, and Laboratory of Magnetism and Superconductivity, Institute of Materials Science, VAST. The authors would like to thank Msc. N.H. Duc, Msc. N.T. Mai, Msc. N.H. Yen and Msc. P.T. Thanh for their help.

\section{REFERENCES}

[1] T. Krenke, E. Duman, M. Acet, E. F. Wassermann, X. Moya, L. Mañosa, and A. Planes, Nature Mater. 4 (2005) 450.

[2] T. Krenke, M. Acet, E. F. Wassermann, X. Moya, L. Mañosa, and A. Planes, Phys. Rev. B 72 (2005) 014412.

[3] P. J. Shamberger, and F. S. Ohuchi, Phys. Rev. B 79 (2009) 144407.

[4] V. V. Khovaylo, K. P. Skokov, O. Gutfleisch, H. Miki, T. Takagi, T. Kanomata, V. V. Koledov, V. G. Shavrov, G. Wang, E. Palacios, J. Bartolomé, and R. Burriel, Phys. Rev. B 81 (2010) 214406.

[5] D. L. Schlagel, W. M. Yuhasz, K. W. Dennis, R. W. McCallum, and T. A. Lograsso, Scripta Mater. 59 (2008) 1083

[6] Y. B. Yang, X. B. Ma, X. G. Chen, J. Z. Wei, R. Wu, J. Z. Han, H. L. Du, C. S. Wang, S. Q. Liu, Y. C. Yang, Y. Zhang, and J. B. Yang, J. Appl. Phys. 111 (2012) 07 A916.

[7] E.C. Passamani, V.P. Nascimento, C. Larica, A.Y. Takeuchi, A.L. Alves, J.R. Provetib, M.C. Pereirac, and J.D. Fabrisd, J. Alloys Comp. 509 (2011) 7826.

[8] D. L. Schlagel, R. W. McCallum, T. A. Lograsso, J. Alloy Compd. 463 (2008) 38.

[9] B. Hernando, J. L. Sánchez Llamazares, J. D. Santos, V. M. Prida, D. Baldomir, D. Serantes, R. Varga, and J. González, Appl. Phys. Lett. 92 (2008) 132507.

[10] I. Babita, S. I. Patil, and S. Ram, J. Phys. D: Appl. Phys. 43 (2010) 205002.

[11] S. E. Muthu, N. V. R. Rao, M. M. Raja, S. Arumugam, K. Matsubayasi, and Y. Uwatoko, J. Appl. Phys. 110 (2011) 083902.

[12] K.G. Sandeman, R. Daou, S. Özcan, J.H. Durrell, N.D. Mathur, and D.J. Fray, Physical Review B 74 (2006) 224436.

[13] K. Morrison, A. Barcza, J.D. Moore, K.G. Sandeman, M.K. Chattopadhyay, S.B. Roy, A.D. Caplin and L.F Cohen, J. Phys. D: Appl. Phys. 43 (2010) 195001.

[14] K. Morrison, Y. Miyoshi, and J.D. Moore, Physical Review B78 (2008) 134418.

[15] L. Mañosa, D. G. Alonso, A. Planes, E. Bonnot, M. Barrio, J. L. Tamarit, S. Aksoy, and M. Acet, Nature 9 (2010) 478.

[16] E. Bonnot, R. Romero, L. Mañosa, E. Vives and Planes, Phys. Rev. Lett. 100 (2008) 125901.

[17] X. Zhou, W. Li, H. P. Kunkel and G. Williams, J. Phys. Condens. Matter 16, (2004) L39.

[18] J. Barth, G. H. Fecher, B. Balke, T. Graf, C. Felser, A. Shkabko and A. Weidenkaff, Phil. Trans. R. Soc. A369 (2011) 3588. 